\title{
An innate talent uncovered
}

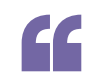

B cells have antibodyindependent protective functions during viral infection

B cells can promote protective immunity to viruses and this has been attributed to their production of high-affinity neutralizing antibodies. A new study by the von Andrian group has challenged this view; it indicates that the ability of B cells to support innate immune responses might be more important than their adaptive immune functions during infection of mice with vesicular stomatitis virus (VSV).

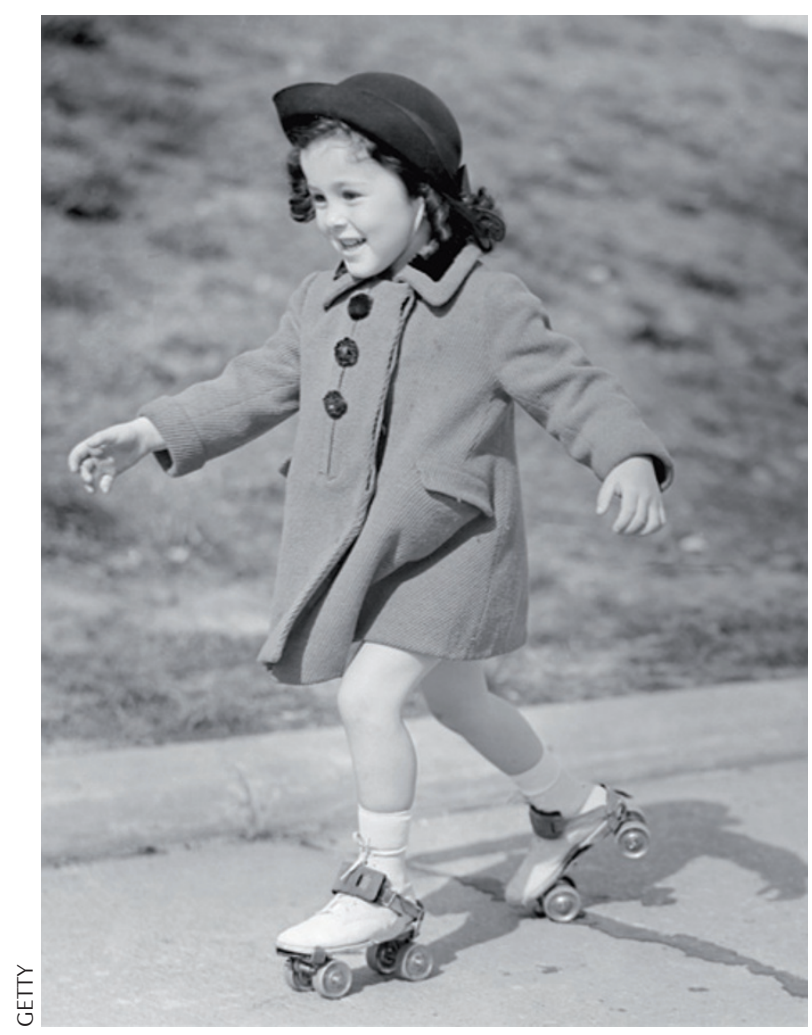

Using a subcutaneous infection protocol - which mimics the natural route of VSV transmission - the authors recently showed that subcapsular sinus (SCS) macrophages in the draining lymph nodes are essential for protective immunity to VSV (see further reading). Productive infection of SCS macrophages and replication of VSV promotes early production of type I interferons (IFNs) and prevents VSV from spreading to the central nervous system. In this study, the authors were interested in defining the role of B cells during VSV infection. They used two transgenic mouse strains: $\mu \mathrm{MT}$ mice, which lack all $B$ cells and have abnormal lymphoid tissue architecture; and $\mathrm{D}_{\mathrm{H}} \mathrm{LMP} 2 \mathrm{~A}$ mice, in which $B$ cells do not express cell-surface B cell receptors and cannot secrete antibodies, but still support normal lymphoid tissue development. Surprisingly, whereas $60 \%$ of $\mu \mathrm{MT}$ mice had fatal neuroinvasion during subcutaneous VSV infection, all of the $\mathrm{D}_{\mathrm{H}} \mathrm{LMP} 2 \mathrm{~A}$ mice were protected. This indicates that $\mathrm{B}$ cells have antibody-independent protective functions during viral infection.

Exploring this further, the authors found that B cells are required to maintain distinct populations of medullary and SCS macrophages in lymph nodes. The lymph nodes of $\mu \mathrm{MT}$ mice contained fewer total macrophages, and $~ 90 \%$ of these macrophages had a medullary phenotype. By contrast, in wildtype or $\mathrm{D}_{\mathrm{H}} \mathrm{LMP} 2 \mathrm{~A}$ mice, only $\sim 40 \%$ of lymph node macrophages had a medullary phenotype, whereas $\sim 60 \%$ had an SCS phenotype. Macrophages from $\mu \mathrm{MT}$ mice could capture and retain lymph-borne VSV in a similar manner to wild-type controls. However, VSV failed to replicate in the $\mu \mathrm{MT}$ macrophages and these cells could not generate a protective IFN response. The ability of B cells to maintain functional lymph node macrophages with a virus-protective SCS phenotype depended on their production of lymphotoxin- $\alpha 1 \beta 2$ (LTa1 $\beta 2)$. When LTa1 $\beta 2$-mediated signalling was blocked in wild-type mice, lymph node macrophages showed aberrant differentiation and were not permissive to VSV replication and IFN induction.

Finally, to assess the contribution of adaptive immune mechanisms other than antibody production, the authors depleted $T$ cells from $\mathrm{D}_{\mathrm{H}} \mathrm{LMP} 2 \mathrm{~A}$ mice before subcutaneous VSV infection. Notably, the mice did not have increased susceptibility to VSV and all survived the infection. These findings indicate that innate immune mechanisms are sufficient to protect the host during VSV infection.

Yvonne Bordon

ORIGINAL RESEARCH PAPER Moseman, E. A et al. B cell maintenance of subcapsular sinus macrophages protects against a fatal viral infection independent of adaptive immunity. Immunity 1 Mar 2012 (doi:10.1016/j.immuni.2012.01.013) FURTHER READING Leavy, O. Antiviral immunity: SCS macrophages protect the CNS. Nature Rev. Immunol. 8, 538 (2010) 